\title{
RKKY range function of a one-dimensional noninteracting electron gas
}

\author{
Gabriele F. Giuliani, ${ }^{1, *}$ Giovanni Vignale, ${ }^{2, \dagger}$ and Trinanjan Datta ${ }^{1}$ \\ ${ }^{1}$ Department of Physics, Purdue University, West Lafayette, Indiana 47907, USA \\ ${ }^{2}$ Department of Physics, University of Missouri, Columbia, Missouri 65211, USA
}

(Received 15 February 2005; published 19 July 2005)

\begin{abstract}
We show that the pitfalls encountered in earlier calculations of the RKKY range function for a noninteracting one-dimensional electron gas at zero temperature can be unraveled and successfully dealt with through a proper handling of the impurity potential.
\end{abstract}

DOI: 10.1103/PhysRevB.72.033411

PACS number(s): 71.10.Ca, 72.10.- d, 71.55.-i, 75.30.Hx

The apparently straightforward evaluation of the Ruderman-Kittel-Kasuya-Yosida (RKKY) range function, or more generally, of the linear density modulation $\delta n(x)$ induced at zero temperature in a noninteracting onedimensional electron gas by a localized static impurity modeled with a $\delta$ function potential has proven surprisingly troublesome. Although the original calculation gave an incorrect answer, ${ }^{1}$ more recent investigations appear to suggest that only a certain procedure, known to lead to a physically sensible answer, should be employed. ${ }^{2}$

The original and most popular procedure is based on the standard theory of linear response. ${ }^{3}$ If the impurity potential is assumed to be of the form $U(x)=\left(\hbar^{2} u / 2 m\right) \delta(x)$ (where $u$ is a suitable wave vector), then one has

$$
\delta n(x)=\frac{\hbar^{2} u}{2 m L} \sum_{q} \chi_{0}(q, 0) e^{i q x},
$$

where $L$ is the system length and $\chi_{0}(q, 0)$ is the static Lindhard response function in one dimension given by

$$
\chi_{0}(q, 0)=\frac{2}{L} \mathcal{P} \sum_{k} \frac{n_{k}-n_{k+q}}{\epsilon_{k+q}-\epsilon_{k}},
$$

where the notation $\mathcal{P}$ stipulates that the principal part of the sum must be taken. Substituting (2) into (1) and using the zero temperature occupation numbers immediately leads to the formula

$$
\delta n(x)=\frac{u}{2 \pi^{2}} \int_{-\infty}^{\infty} d q e^{i q x} \int_{-k_{F}}^{k_{F}} d k\left(\frac{1}{2 k q+q^{2}}-\frac{1}{2 k q-q^{2}}\right) .
$$

The interpretation and handling of this expression rest at the origin of the problem at hand.

If one simply proceeds to explicitly evaluate the integral over $k$ in Eq. (3), or, which is the same, that of (2), the result is well known and is given by

$$
\chi_{0}(q, 0)=\frac{2 m}{\pi \hbar^{2} q} \ln \left|\frac{2 k_{F}+q}{2 k_{F}-q}\right| .
$$

At this point the second integral over $q$ can be readily evaluated leading to

$$
\delta n(x)=-\frac{u}{\pi} \operatorname{si}\left(2 k_{F} x\right)
$$

where the sine integral function appears. ${ }^{4}$ This result provides the correct answer to the problem. In particular, the large distance behavior turns out to be

$$
\delta n(x) \simeq-\frac{u}{\pi} \frac{\cos \left(2 k_{F} x\right)}{x}
$$

an expression displaying the expected decay and Friedel oscillations. ${ }^{5}$ One should note at this point that, in view of the singular behavior of the integrand at the origin, however tempting, the order of the $k$ and $q$ integrations in Eq. (3) cannot be freely exchanged. ${ }^{2}$ Doing so leads to the manifestly unphysical answer ${ }^{1}$

$$
\delta n(x)=-\frac{u}{\pi}\left(\operatorname{si}\left(2 k_{F} x\right)+\frac{\pi}{2}\right) \quad(\text { not! })
$$

This pitfall is unique to the one-dimensional case for in two and three dimensions, formulas equivalent to (3) can be derived and safely evaluated by exchanging at will the order of the intervening integrations.

An alternative, appealing and equally physically valid procedure to obtain $\delta n(x)$ is in the case offered by extracting the leading linear term of the formula

$$
n(x)-n_{0}=2 \sum_{|\vec{k}| \leqslant k_{F}}\left(\left|\psi_{k}(x)\right|^{2}-\left|\psi_{k}^{(0)}(x)\right|^{2}\right),
$$

valid for single Slater determinant states. Now, if the usual first-order perturbation theory result for $\psi_{k}(x)$ is used in (8), one is immediately led to an equation that differs from (3) merely for the exchange of the order of the quadratures. ${ }^{1}$ Since, as observed above, such an expression does lead to an unphysical result, it has been suggested that this route is unphysical and should be avoided. ${ }^{2}$ We find that this conclusion is unwarranted for, as we show next, the difficulty lies here with the use of perturbation theory, which is invalid, and not with Eq. (8) per se.

To prove our assertion we observe that the exact delocalized eigenstates of the Schrödinger equation 


$$
\frac{\hbar^{2}}{2 m}\left\{-\frac{\partial^{2}}{\partial x^{2}}+u \delta(x)\right\} \psi(x)=E \psi(x),
$$

can be written as a superposition of the two following (normalized) scattering states

$$
\psi_{k+}(x)= \begin{cases}\frac{e^{i k x}}{\sqrt{L}}+\frac{u}{2 i k-u} \frac{e^{-i k x}}{\sqrt{L}}, & x<0 \\ \frac{2 i k}{2 i k-u} \frac{e^{i k x}}{\sqrt{L}}, & x>0\end{cases}
$$

and

$$
\psi_{k-}(x)= \begin{cases}\frac{2 i k}{2 i k-u} \frac{e^{-i k x}}{\sqrt{L}}, & x<0 \\ \frac{e^{-i k x}}{\sqrt{L}}+\frac{u}{2 i k-u} \frac{e^{i k x}}{\sqrt{L}}, & x>0,\end{cases}
$$

where $k$ is limited to positive values only. By adding the modulus squares of $\psi_{k+}(x)$ and $\psi_{k-}(x)$ and by summing over $k>0$ in (8), one obtains the following exact expression for the electronic density $n(x)$ :

$$
n(x)=\frac{2}{\pi} \int_{0}^{k_{F}} d k\left(1+\frac{2 u k \sin (2 k|x|)}{4 k^{2}+u^{2}}-\frac{u^{2} \cos (2 k|x|)}{4 k^{2}+u^{2}}\right) .
$$

At this point we are left with extracting the linear term in $u$. This must be done with some care. In particular, one must resist the temptation to simply drop the last term in Eq. (12) and, at the same time, to neglect the $u^{2}$ in the denominator of the second one. To do so coincides with making use of firstorder perturbation theory and leads exactly to the original unphysical result of Eq. (7). Although it is safe to handle the second term of (12) as just described, the third term does contribute a first-order term that can be readily extracted by making use of the relation

$$
\lim _{u \rightarrow 0} \frac{u}{4 k^{2}+u^{2}}=\frac{\pi}{2} \delta(k)
$$

leading to

$$
n(x) \simeq \frac{2 k_{F}}{\pi}+\frac{u}{\pi}\left(\int_{0}^{k_{F}} d k \frac{\sin (2 k|x|)}{k}-\frac{\pi}{2}\right),
$$

a result that can be readily seen to coincide with the correct answer of Eq. (5).

We have therefore shown that proper handling of the impurity potential allows one to correctly carry out the calculation of the linear density modulation via either the response function method of Eq. (1) or the alternative direct procedure offered by (8). ${ }^{6}$

We conclude by remarking that a proper treatment of the problem of the effects of a static localized impurity in an interacting one-dimensional electron liquid can be achieved by means of the Luttinger liquid model. The problem is highly nontrivial as, although Friedel-like oscillations with an amplitude decay generally ruled by the interaction strength exist at intermediate distances, ${ }^{3}$ for large distances the physics of the phenomenon is nonlinear in the impurity potential. ${ }^{7}$ Finally, a recent discussion of the effects of the Coulomb interaction on the Friedel oscillations of an electron liquid in two and three dimensions can be found in Ref. 8.

The authors wish to thank A. W. Overhauser and George E. Simion for useful conversations.
*Electronic address: gfg@physics.purdue.edu, tdatta@physics.purdue.edu

†Electronic address: vignaleg@missouri.edu

${ }^{1}$ C. Kittel, in Solid State Physics, vol. 22, edited by F. Seitz, D. Turnbull, and H. Ehreinreich (Academic Press, New York, 1968) see also erratum ibidem.

${ }^{2}$ Y. Yafet, Phys. Rev. B 36, 3948 (1987).

${ }^{3}$ G. F. Giuliani and G. Vignale, Quantum Theory of the Electron Liquid (Cambridge University Press, Cambridge, England, 2005).
${ }^{4}$ I. Gradshteyn and I. Ryzhik, Table of Integrals, Series and Products (Academic Press, San Diego, 1965).

${ }^{5}$ J. Friedel, Adv. Phys. 3, 446 (1954).

${ }^{6}$ Of course the correct answer can also be arrived at by calculating $\delta n(x)$ at finite temperature and then taking the $T \rightarrow 0$ as done for instance in V. I. Litvinov and V. K. Dugaev, Phys. Rev. B 58, 3584 (1998). This sheds no light onto the problem at hand.

${ }^{7}$ R. Egger and H. Grabert, Phys. Rev. Lett. 75, 3505 (1995).

${ }^{8}$ G. Simion and G. F. Giuliani Phys. Rev. B 72, 045127 (2005). 